\title{
A Transmit Beamforming and Nulling Approach with Distributed Scheduling to Improve Cell Edge Throughput
}

\author{
Wendy C. Wong, Qinghua Li, and Shilpa Talwar \\ Intel Labs, Intel Corporation, 2200 Mission College Boulevard, Santa Clara, CA 95054, USA \\ Correspondence should be addressed to Wendy C. Wong, wendy.c.wong@intel.com
}

Received 16 September 2009; Accepted 16 December 2009

Academic Editor: Hongxiang Li

Copyright ( $) 2010$ Wendy C. Wong et al. This is an open access article distributed under the Creative Commons Attribution License, which permits unrestricted use, distribution, and reproduction in any medium, provided the original work is properly cited.

We propose a transmit scheme for WiMAX systems, where multiple base stations (BSs) employ downlink transmit beamforming and nulling for interference mitigation, with minimal coordination amongst BSs. This scheme improves system throughput and robustness, by increasing cell edge and overall cell throughputs by $68 \%$ and $19 \%$, respectively, and by delivering improvement for mobile speed up to $60 \mathrm{~km} / \mathrm{h}$. First, cell edge users suffering from severe interferences are identified. Next, the RRM unit allocates resource to serving cell edge users only. BSs will schedule to serve their cell edge users independently using the allocated resources by the RRM. A special uplink sounding region is designed for BSs to learn the interference environment and form proper beams and nulls. The nulls formed towards users served by other BSs reduced interference from a BS towards these users and is the basic building block of our algorithm.

\section{Introduction}

In a cellular network with frequency reuse one, downlink (DL) performance is limited by cochannel interference. In the downlink of a cellular system, it is well known that BS with multiple transmit antennas can improve the desired signal power by transmit beamforming [1-3]. However, the performance gain generated by the nulls from the multiple antennas is much less studied.

In Figure 1, BS $i$ helps BS $j$ by forming a null towards MS $j$ and BS $j$ helps BS $i$ by forming a null towards MS $i$. Hence, the SINR at MS $i$ and MS $j$ is increased by higher signal power from the beam of its own serving BS and reduced interference from the null of a nearby interfering BS. This is referred to as beamforming and nulling (BFaN) from hereon. All BSs with cell edge MSs must enable BFaN simultaneously in order to achieve throughput improvement for all cell edge MSs. For example, if $\mathrm{BS} i$ enables $\mathrm{BFaN}$ while BS $j$ does not, only MS $j$ benefits from the reduced interference. Note that MS $i$ denotes the cell edge MS that is currently being scheduled to be served by BS $i$. Hence, in the next frame, another cell edge MS may be scheduled and MS $i$ will refer to a different cell edge MS. However, we keep the same MS index to simplify our notations. In addition, $\mathrm{BFaN}$ at the BS is attractive since it moves the implementation and computation complexity from MSs (which is more cost sensitive and power limited) to BSs.

This paper studies the benefits of $\mathrm{BFaN}$ and has made two key contributions. First, a simple and effective BFaN scheme is proposed for cellular systems with multiple BS antennas. Unlike conventional downlink beamforming with nulling schemes, our BFaN scheme eliminates the restriction for equal UL and DL resource allocation by using a special UL sounding mechanism. Second, it quantifies the performance gains of $\mathrm{BFaN}$ in realistic cellular settings. It should be noted that the system throughput gains cannot be observed from link level simulations that are only suitable for a point to point setting due to the dynamic interference environment created by BS scheduling. System level simulations (SLSs) with multiple scheduled links in multi-cells and multisectors are required. This may be the reason for the lack of research results reported in the literature.

The paper is divided into literature survey, system model description, our proposed $\mathrm{BFaN}$ scheme, simulation results, and conclusions. The literature survey includes other interference mitigation techniques considered in IEEE $802.16 \mathrm{~m}$ 


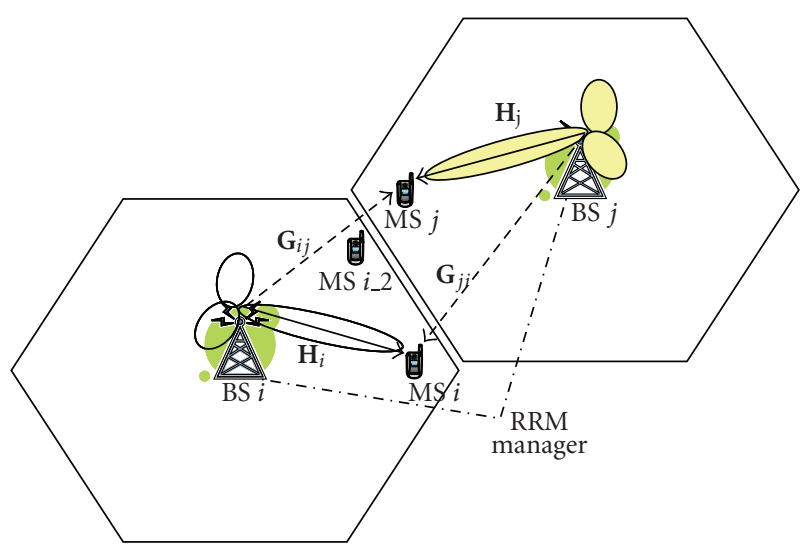

Figure 1: A simple 2 cell deployment showing BFaN.

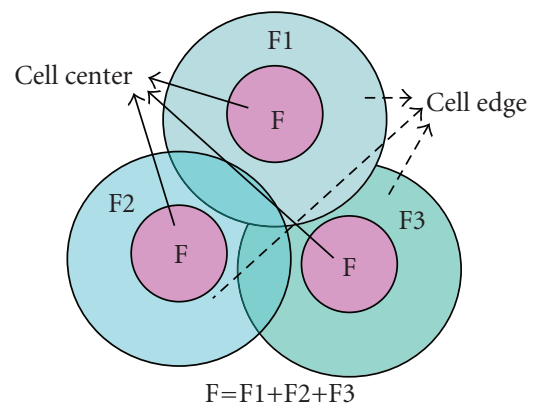

Figure 2: Vanilla FFR scheme.

study group. Most of these techniques are developed within the past year.

\section{Literature Survey}

In this section, we present a survey of schemes that were recently proposed by IEEE $802.16 \mathrm{~m}$ task groups in [4] for interference mitigation in frequency reuse one systems. They include fractional-frequency reuse (FFR), precoding matrix index (PMI) restriction, multi-BS MIMO, interference mitigation using power control and scheduling, and multi-hop relay. These not only provide a background and reference for our scheme but are of interest to readers because most of them were published within the past year.

2.1. FFR. A system with FFR employs multiple frequency reuse factors within a cell, for example, reuse 1 and 3, as depicted in Figure 2. At the cell center where interference signal is weak, each BS can use frequency reuse 1. In contrast, at the cell edge where interference is strong, each BS only uses a portion of the bandwidth, for example, using reuse 3. Assuming that users are uniformly distributed throughout the network, the cumulative distribution function of users' average SINR shows that in a reuse 1 network more than $30 \%$ users' average SINR is below 0dB. Such users are typically at cell edge, and will likely experience a poor network connection, low downlink throughput, and high probability of outage. Higher frequency reuse factors, such as 3, can significantly reduce the cochannel interference amongst neighboring cells/sectors in that $2 / 3$ of the cochannel interference sources are eliminated compared with reuse 1 networks. This leads to greatly improved coverage and average SINR for cell edge users. The gain from FFR comes from the reduced interference level to cell edge users. However, improvement of downlink average SINR by using higher reuse factors is achieved at the cost of system spectrum efficiency, defined as the ratio of system throughput to occupied spectrum bandwidth, since higher reuse also requires more spectrum bandwidth. One main advantage of FFR over other schemes is its ability to support users with high mobility. Hence, FFR is naturally complementary to our $\mathrm{BFaN}$ scheme, which primarily supports low to moderate speed mobile users.

2.2. PMI Restriction for Downlink Closed-Loop MIMO. The precoding matrix index (PMI) restriction scheme $[5,6]$ sacrifices cell center performance to reduce interference experienced by cell edge MSs. On a periodic basis, cell edge MSs measure the interference experienced from neighboring BSs transmission to their cell center MSs. The MSs report to their own serving BS the PMIs that cause high interference and these PMIs will be excluded from the original beamforming codebook. BSs will exchange the list of excluded PMIs over the backhaul. As a result, cell center MSs are served using a subset of the original beamforming codebook while cell edge MSs are served with the complete codebook. Namely, BSs may need to use precoding matrices that are not optimal for its transmission to cell center users. Hence, cell center throughput drops while cell edge throughput gains.

2.3. Multi-BS MIMO. In multi-BS MIMO, cell edge MSs in multiple cells can be served jointly and simultaneously by multiple BSs using the same channel resources. The multiple BSs act as a single BS with distributed antennas at the physical layer. However, this scheme increases system implementation cost significantly due to increased co-ordination. First, BSs need to have access to data destined for MSs of other BSs for macro-diversity. Second, a flexible pilot pattern design with a configurable pilot reuse factor depending on the number of BSs participating in the system is needed for accurate channel measurements and beamforming weight calculation. Third, scheduling decision will be jointly made at the BSs. Performance gain achieved by multi-BS MIMO in [7] for cell edge MSs at $30 \mathrm{~km} / \mathrm{h}$ is modest at $26.14 \%$ while the overall gross throughput rate actually dropped by $1.5 \%$. Hence, with all the increased complexities and BS cooperation, the gain of this scheme is moderate.

\subsection{Interference Mitigation by Power Control and Scheduling.} A base station can choose to boost its DL transmit power to selected cell edge MSs. However, the boosted signal transmissions will cause stronger interference to cell edge MSs of neighboring cells. As an alternative, a BS can coordinate with other BSs to schedule MSs with high mutual 
interference potential on different channel resources [4]. However, this requires periodic BS coordination. Again, since BS operators may not have control over the backhaul latency, this scheme may not be implemented when the backhaul latency exceeds the latency requirement.

2.5. Multihop Relay. The original idea of multi-hop relay is to extend the BS coverage to isolated pockets within or outside the current BS coverage area on a temporary (for emergency response) or permanent basis. The presence of a relay can potentially help cell edge MSs. However, in-band relays operating in a time sharing fashion will decrease system peak throughput and the decrease is scaled down by the number of hops. Hence, relay deployment decreases spectrum efficiency and increases system overheads. For instance, additional preambles in the PHY layer and additional MAC signaling overheads (e.g., dedicated relay zone allocation) are needed to maintain the multi-hop links. Out-of-band relays will increase BS and MS production cost since these devices will need to support multiple radio bands in order to communicate with these relays. Finally, not all operators will deploy relays and hence we cannot count on relay as a solution to improve cell edge user throughput.

\section{System Model and Beamforming Weight Calculation}

We consider a time division duplex (TDD) system where the uplink (UL) and downlink (DL) channels are transposes of each other due to channel reciprocity. It is assumed that each BS has $M$ antennae while each SS has $N$ antennae.

The DL transmit and UL receive system models are introduced in Sections 3.1 and 3.2. Next, detailed beamforming weight calculation at BSs and MSs is described in Sections 3.3-3.5. Assume that the cell edge users transmitting information to their serving BSs in the UL will be allocated the same resources in the DL transmission from their BSs, UL beamforming weights applied at their BSs to receive information can be used in the DL to transmit information to the same set of cell edge users. Hence, our beamforming weight calculation process starts with looking at the UL beamforming weight calculation at each BS in Section 3.3. Next we show how to derive an optimal DL beamforming weight aiming to maximize cell edge user SINR and how this DL beamforming weight can be approximated by the normalized complex conjugate of the UL beamforming weight in Section 3.4. Last, the DL beamforming weight at a cell edge MS is shown in Section 3.5. Ideally, beamforming weight should be calculated for each subcarrier. However, to reduce overhead, pilots that are used for beamforming weight calculation are scattered over the whole allocated resources. Beamforming weights for intermediate subcarriers can be interpolated from beamforming weights calculated from these pilots in many different ways.

3.1. Data Model for BS DL Transmit. Consider the system model depicted in Figure 3 in the DL (from BSs to MSs)

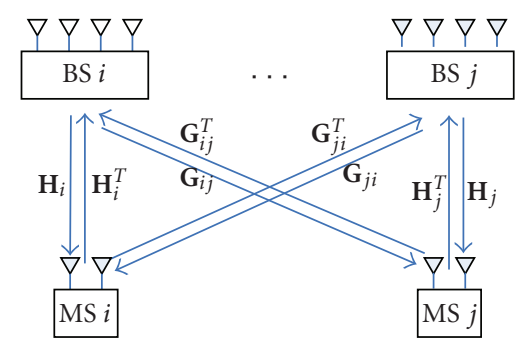

FIgURe 3: BFaN system model.

direction, the received signal at MS $i$ is

$$
\begin{aligned}
\mathbf{x}_{\mathrm{MS} i}= & \mathbf{H}_{i} \mathbf{w}_{\mathrm{DL} i} s_{\mathrm{BS} i} \\
& +\sum_{\substack{j, j \neq i \text { all interfering } \\
\text { neighboring BS }}} \mathbf{G}_{j i} \mathbf{w}_{\mathrm{DL} j} s_{\mathrm{BS} j}+\mathbf{n}_{\mathrm{MS}},
\end{aligned}
$$

where $\mathbf{H}_{i}$ is the $N \times M$ channel matrix from serving BS $i$ to MS $i ; \mathbf{w}_{\mathrm{DL} i}$ and $\mathbf{w}_{\mathrm{DL} j} \mathrm{~s}$ are DL transmit beamforming vectors applied by BS $i$ and BS $j$, respectively; $\mathbf{G}_{j i}$ is the $N \times M$ interfering channel matrix from BS $j$ to MS $i$; $\mathbf{n}_{\mathrm{MS}}$ is the noise vector at MSs. Note that (1) describe the received signal vector at MS $i$ for each subcarrier. However, the subcarrier index is usually dropped to simplify notation.

3.2. Data Model for BS UL Receive. Consider the system model in the UL direction in Figure 3, the received signal at BS $i$ is

$$
\mathbf{x}_{i}=\mathbf{H}_{i}^{T} \mathbf{w}_{\mathrm{MS} i} s_{i}+\sum_{\substack{j, j \neq i, \text { cell edge users } \\ \text { of all interfering BSs }}} \mathbf{G}_{i j}^{T} \mathbf{w}_{\mathrm{MS} j} s_{j}+\mathbf{n},
$$

where $\mathbf{H}_{i}^{T}$ is the $M \times N$ channel matrix from MS $i$ to its serving $\mathrm{BS} i ; \mathbf{w}_{\mathrm{MS} i}$ and $\mathbf{w}_{\mathrm{MS} j}$ are the transmit beamforming vectors at MSs $i$ and $j$, respectively; $s_{i}$ and $s_{j}$ are the transmitted QAM symbols from MSs $i$ and $j$, respectively; $\mathrm{G}_{i j}^{T}$ is the $N \times M$ interfering channel matrix for MS $j$ to BS $i$; $\mathbf{n}$ is the noise vector at BSs.

If an MS can transmit using $N(N>1)$ antennae in the UL, it can set its BF weight using various methods. For example, if an MS can only transmit using 1 antenna in the $\mathrm{UL}, \mathbf{w}_{\mathrm{MSi}}(k)=[1,0, \ldots, 0]^{T}$. If an MS can transmit using all $N$ antennae, $\mathbf{w}_{\mathrm{MS} i}=\mathbf{v}_{1}$ where $\mathbf{v}_{1}(k)(N \times 1)$ can be the right singular vector corresponding to the largest singular value of $\mathbf{H}_{i}^{T}[8]$.

3.3. UL BS Beamforming Weight Computation. Let $\mathbf{w}_{i}(M \times$ 1) denote the UL beamforming weight vector at BS $i$. The 


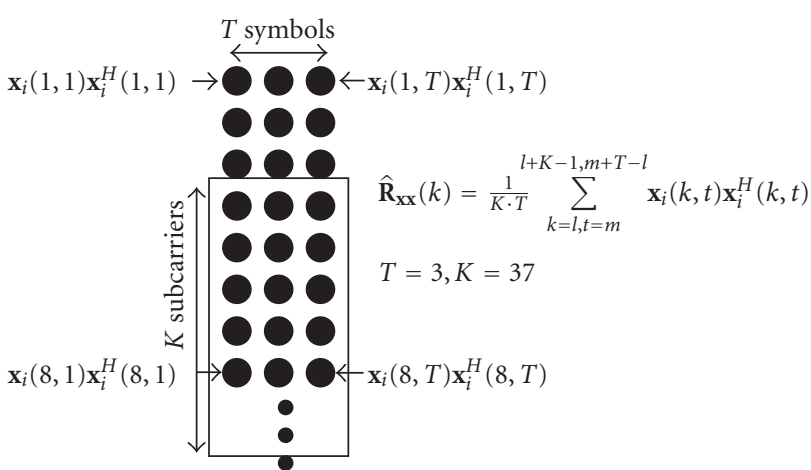

FIGURE 4: Moving average window.

estimated signal after applying UL BS beamforming weight on the UL receive signal in (2) becomes

$$
\begin{aligned}
\widehat{s}_{\mathrm{i}}= & \mathbf{w}_{i}^{H} \mathbf{x}_{i} \\
= & \mathbf{w}_{i}^{H} \mathbf{H}_{i}^{T} \mathbf{w}_{\mathrm{MS} i} s_{i} \\
& +\sum_{\substack{j, j \neq i, \text { cell edge users } \\
\text { of all interfering BSs }}} \mathbf{w}_{i}^{H} \mathbf{G}_{i j}^{T} \mathbf{w}_{\mathrm{MS} j} s_{j}+\mathbf{w}_{i}^{H} \mathbf{n} . \\
& \sum
\end{aligned}
$$

The beamforming weight vector $\mathbf{w}_{i}$ is derived by minimizing the mean squared error (MMSE) $E\left(\left|\hat{s}_{\mathrm{MS} i}-s_{\mathrm{MS} i}\right|^{2}\right)$ and is

$$
\begin{aligned}
\mathbf{w}_{i} & =\mathbf{R}_{\mathbf{x} \mathbf{x}_{i}}^{-1} \mathbf{H}_{i}^{T} \mathbf{w}_{\mathrm{MS} i}, \\
\mathbf{R}_{\mathbf{x x}_{i}} & =E\left(\mathbf{x}_{i} \mathbf{x}_{i}^{H}\right) .
\end{aligned}
$$

At each BS, a moving window averaging technique in Figure 4 is used to generate a covariance matrix estimate $\hat{\mathbf{R}}_{\mathbf{x x}_{i}}(k)$ for each subcarrier $k$. The averaging is performed over frequency and time assuming the channel response during the frequency and time span is similar. We have investigated adding smoothing techniques [9] or Cholesky decomposition plus smoothing [10] for improving the accuracy of covariance matrix estimation. However, the performance gain is negligible.

Next, each BS can measure $\mathbf{H}_{i}^{T} \mathbf{w}_{\mathrm{MS} i}$ from its uplink received signal. Therefore, each $\mathrm{BS}$ will be able to calculate its UL beamforming weight using (4).

Assuming noise and data are uncorrelated and data from various MSs are uncorrelated, the covariance matrix in (5) can be expanded as

$$
\begin{aligned}
\mathbf{R}_{\mathbf{x x} i}= & \sigma_{s}^{2} \mathbf{H}_{i}^{T} \mathbf{w}_{\mathrm{MS} i} \mathbf{w}_{\mathrm{MS} i}^{H}\left(\mathbf{H}_{i}^{T}\right)^{H} \\
& +\sigma_{s}^{2} \sum_{\substack{i \neq j, \text { cell edge users } \\
\text { of all interfering BSs }}} \mathbf{G}_{i j}^{T} \mathbf{w}_{\mathrm{MS} j} \mathbf{w}_{\mathrm{MS} j}^{H}\left(\mathbf{G}_{i j}^{T}\right)^{H}+\sigma^{2} \mathbf{I} .
\end{aligned}
$$

However, in the covariance matrix approximation process, there may be non zero cross terms shown in (A.1) in the appendix. These cross terms will cause inaccurate covariance matrix estimation and cause system degradation. Hence, to compare the performance degradation caused by inaccurate covariance matrix estimation, we have implemented the reference scheme in our simulation. In the reference scheme, BSs first schedule their cell edge MSs over the allocated resources independently and then calculate the covariance matrix using (6) and beamforming weights assuming perfect channel knowledge using (4). To mimic the delay in backhaul scheduling information exchange, the scheduling decision is made with perfect channel knowledge that exists $5 \mathrm{~ms}$ earlier.

3.4. DL BS Beamforming Weight Computation. Consider only the DL transmission from BS $i$ to MS $i$, the receive signal at cell edge MS $1,2, \ldots, i, \ldots, N_{\mathrm{BS}}$ is a vector. If the DL transmit beamforming weight vector at BS $i$ and the MSs' receive beamforming vector ( $\mathbf{w}_{\mathrm{MS} i \mathrm{DL}}$ ) have been set correctly, the following expression will be minimized ignoring the noise term (since DL is interference limited):

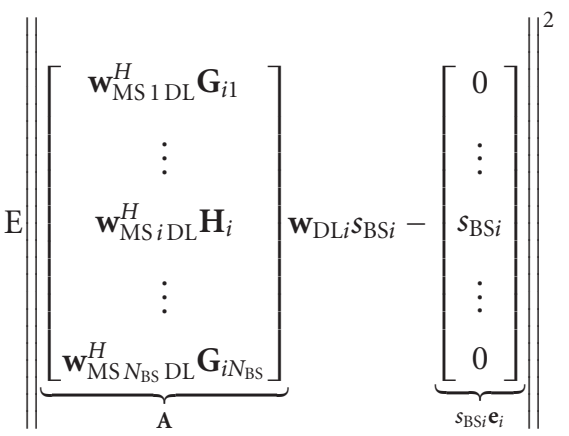

$$
\begin{aligned}
& =\mathrm{E}\left\|\mathrm{Aw}_{\mathrm{DL} i} s_{\mathrm{BS} i}-s_{\mathrm{BS} i} \mathbf{e}_{i}\right\|^{2} \\
& =\mathrm{E}\left(\left(\mathbf{w}_{\mathrm{DL} i}^{H} \mathbf{A}^{H} \mathbf{A} \mathbf{w}_{\mathrm{DL} i}-\mathbf{w}_{\mathrm{DL} i}^{H} \mathbf{A}^{H} \mathbf{e}_{i}-\mathbf{e}_{i}^{H} \mathbf{A} \mathbf{w}_{\mathrm{DL} i}+1\right) s_{\mathrm{BS} i}^{*} s_{\mathrm{BS} i}\right),
\end{aligned}
$$

where $\mathbf{A}$ is $N_{\mathrm{BS}} \times M$ matrix, $\mathbf{e}_{i}\left(N_{\mathrm{BS}} \times 1\right)$ has all zero entries except a one at the $i$ th position; $N_{\mathrm{BS}}$ is the number of BSs participating in the beamforming and nulling. Taking derivative of (7) with respect to $\mathbf{w}_{\mathrm{DL} i}$ and setting the derivative to zero, we have

$$
\begin{aligned}
\mathbf{w}_{\mathrm{DL} i}^{H} \mathrm{E}\left(\mathbf{A}^{H} \mathbf{A} s_{\mathrm{BS} i}^{*} s_{\mathrm{BS} i}\right) & =\mathrm{E}\left(\mathbf{e}_{i}^{H} \mathbf{A}\right) \\
& \Longrightarrow \mathrm{E}\left(\mathbf{A}^{H} \mathbf{A} s_{\mathrm{BS} i}^{*} s_{\mathrm{BS} i}\right) \mathbf{w}_{\mathrm{DL} i}=\mathrm{E}\left(\mathbf{A}^{H} \mathbf{e}_{i}\right) \\
\mathbf{w}_{\mathrm{DL} i} & =\left(\mathrm{E}\left(\mathbf{A}^{H} \mathbf{A} s_{\mathrm{BS} i}^{*} s_{\mathrm{BS} i}\right)\right)^{-1} \mathrm{E}\left(\mathbf{A}^{H} \mathbf{e}_{i}\right) \\
& =\mathbf{R}_{i}^{-1} \mathbf{H}_{i}^{H} \mathbf{w}_{\mathrm{MS} i \mathrm{DL}}
\end{aligned}
$$

where

$$
\begin{aligned}
\mathbf{R}_{i}= & \mathrm{E}\left(\mathbf{A}^{H} s_{\mathrm{BS} i}^{*} s_{\mathrm{BS} i} \mathbf{A}\right) \\
= & \mathbf{H}_{i}^{H} \mathbf{w}_{\mathrm{MS} i \mathrm{DL}} \mathbf{w}_{\mathrm{MS} i \mathrm{DL}}^{H} \mathbf{H}_{i} \sigma_{\mathrm{BS}}^{2} \\
& +\sigma_{\mathrm{BS}}^{2} \sum_{j, j \neq i} \mathbf{G}_{i j}^{H} \mathbf{w}_{\mathrm{MS} j \mathrm{DL}} \mathbf{w}_{\mathrm{MS} j \mathrm{DL}}^{H} \mathbf{G}_{i j} .
\end{aligned}
$$


Setting $\mathbf{w}_{\mathrm{MS} i \mathrm{DL}}=\mathbf{w}_{\mathrm{MS} i}^{*}$ in (10), and take the complex conjugate of (10), we have

$$
\begin{aligned}
\mathbf{R}_{i}^{*}= & \mathbf{H}_{i}^{T} \mathbf{w}_{\mathrm{MS} i \mathrm{DL}}^{*} \mathbf{w}_{\mathrm{MS} i \mathrm{DL}}^{T} \mathbf{H}_{i}^{*} \sigma_{\mathrm{BS}}^{2} \\
& +\sum_{j, j \neq i} \mathbf{G}_{i j}^{T} \mathbf{w}_{\mathrm{MS} j \mathrm{DL}}^{*} \mathbf{w}_{\mathrm{MS} j \mathrm{DL}}^{T} \mathbf{G}_{i j}^{*} \sigma_{\mathrm{BS}}^{2} \\
= & \mathbf{H}_{i}^{T} \mathbf{w}_{\mathrm{MS} i} \mathbf{w}_{\mathrm{MS} i}^{H} \mathbf{H}_{i}^{*} \sigma_{\mathrm{BS}}^{2}+\sum_{j, j \neq i} \mathbf{G}_{i j}^{T} \mathbf{w}_{\mathrm{MS} j} \mathbf{w}_{\mathrm{MS} j}^{H} \mathbf{G}_{i j}^{*} \sigma_{\mathrm{BS}}^{2} .
\end{aligned}
$$

Assuming $\sigma_{s}^{2}=\sigma_{\mathrm{BS}}^{2}$ (since power of QAM symbols are usually normalized to 1) and equal UL and DL resource allocation (this implies that the same MSs that tranmit in the UL will be receiving from their serving BSs in the DL and the BS and MS indexes will be referring to the same BSs and MSs in both UL and DL), combining (6) and (11), we have $\mathbf{R}_{\mathbf{x x}_{i}}=\mathbf{R}_{i}^{*}+\sigma^{2} \mathbf{I} \approx$ $\mathbf{R}_{i}^{*}$ since BSs usually have low noise figure and low thermal noise. From (9), we have

$$
\begin{aligned}
\mathbf{w}_{\mathrm{DL} i} & =\mathbf{R}_{i}^{-1} \mathbf{H}_{i}^{H} \mathbf{w}_{\mathrm{MS} i \mathrm{DL}} \\
& =\mathbf{R}_{i}^{-1} \mathbf{H}_{i}^{H} \mathbf{w}_{\mathrm{MS} i}^{*}=\left(\left(\mathbf{R}_{i}^{*}\right)^{-1} \mathbf{H}_{i}^{T} \mathbf{w}_{\mathrm{MS} i}\right)^{*} \\
& \approx\left(\left(\mathbf{R}_{\mathbf{X x}}\right)^{-1} \mathbf{H}_{i}^{T} \mathbf{w}_{\mathrm{MS} i}\right)^{*}=\mathbf{w}_{i}^{*} .
\end{aligned}
$$

Hence, the optimal DL BS beamforming weight is derived to be the complex conjugate of the BS UL beamforming weight. The actual BS DL beamforming weight used is

$$
\mathbf{w}_{\mathrm{DL} i}=\frac{\mathbf{w}_{i}^{*}}{\left\|\mathbf{w}_{i}\right\|} .
$$

The normalization is used to ensure that applying the DL BS beamforming weight to the signal will not change its power level. For TDD systems, instead of calculating a separate DL BS beamforming weight, the DL BS beamforming weight can be set to be the normalized conjugated BS UL beamforming weight in (13) assuming equal UL and DL resource allocation.

3.5. Calculation of Receive Beamforming Weight at MS. If MS $i$ has $N(N>1)$ antennae, it can apply beamforming and nulling at its DL receiver to further improve its SINR. Note that this is a deviation from the assumption made in DL BS beamforming calculation. However, the degradation is minimum as shown by our simulation results reported in Section 4. At MS $i$, the estimated DL receive signal after applying beamforming and nulling to signal in (1) is

$$
\begin{aligned}
\hat{s}_{\mathrm{BS} i}= & \mathbf{w}_{\mathrm{MS} i \mathrm{DL}}^{H} \mathbf{x}_{\mathrm{MS} i} \\
= & \mathbf{w}_{\mathrm{MS} i \mathrm{DL}}^{H} \mathbf{H}_{i} \mathbf{w}_{\mathrm{DL} i} s_{\mathrm{BS} i} \\
& +\sum_{\substack{j, j \neq i \text { all interfering } \\
\text { neighboring BS }}}^{\mathbf{w}_{\mathrm{MS} i \mathrm{DL}}^{H} \mathbf{G}_{j i} \mathbf{w}_{\mathrm{DL} j} s_{\mathrm{BS} j}+\mathbf{w}_{\mathrm{MS} i \mathrm{DL}}^{H} \mathbf{n}_{\mathrm{MS}} .}
\end{aligned}
$$

The MS beamforming weight $\mathbf{w}_{\mathrm{MS} i \mathrm{DL}}(N \times 1)$ is derived by minimizing the mean squared error $E\left(\left|\hat{s}_{\mathrm{BS} i}-s_{\mathrm{BS} i}\right|^{2}\right)$ and is

$$
\begin{aligned}
\mathbf{w}_{\mathrm{MS} i \mathrm{DL}} & =\mathbf{R}_{\mathbf{x}_{\mathrm{MS} i} \mathbf{x}_{\mathrm{MS} i}}^{-1} \mathbf{H}_{i} \mathbf{w}_{\mathrm{DL} i} \\
& =\mathbf{R}_{\mathbf{x}_{\mathrm{MS} i} \mathbf{x}_{\mathrm{MS} i}}^{-1} \mathbf{H}_{i} \frac{\mathbf{w}_{i}^{*}}{\left|\mathbf{w}_{i}\right|}, \\
\mathbf{R}_{\mathbf{x}_{\mathrm{MS} i} \mathbf{x}_{\mathrm{MS} i}} & =E\left(\mathbf{x}_{\mathrm{MS} i} \mathbf{x}_{\mathrm{MS} i}^{H}\right) .
\end{aligned}
$$

Again, $\mathbf{H}_{i}\left(\mathbf{w}_{i}^{*} /\left|\mathbf{w}_{i}\right|\right)$ can be measured by pilots in the DL transmission and $\mathbf{R}_{\mathbf{x}_{\mathrm{MSi}} \mathbf{x}_{\mathrm{MS} i}}$ can be estimated by using the received data depicted in (1) and using the same moving average method depicted in Figure 4.

\section{Proposed BFaN Scheme}

In conventional downlink transmit beamforming and nulling scheme for TDD systems, each BS derives a UL receive beamforming weight using signal received in the UL. Assume equal UL and DL resource allocation, each BS can use the normalized conjugated UL receive beamforming weight in (13) as its DL transmit beamforming weight. Compared to FDD or to system deploying DL beamforming alone, this saves channel feedback overhead and avoids channel information exchange among BSs/MSs. In addition, it is more responsive to channel exchange because it avoids the channel feedback latency. However, the restriction of equal UL and DL resource allocation is not practical and generates waste in using channel resource due to the uneven UL and DL traffic pattern. Traffic is much heavier in the DL than UL. It is highly likely to have DL traffic destined to a MS but the MS does not have any UL traffic. Hence, allocating resource for that user in the UL and ask it to just send some junk data to enable the beamforming weight calculation will deprive another user to use the UL transmission opportunity. To remove this restriction on resource allocation and eliminate waste while at the same time taking advantages offered by this scheme, we propose our BFaN to increase cell edge user throughput.

Our $\mathrm{BFaN}$ scheme is comprised of 3 steps:

(1) cell edge MS identification,

(2) resource allocation for cell edge MSs,

(3) distributed scheduling.

Our BFaN scheme provides two major advantages over previous interference mitigation schemes. First, it uses existing feedback and UL sounding mechanisms in existing IEEE 802.16 systems. Hence, this scheme can be implemented without additional standard changes. Second, no real time BS collaboration and real time information exchange over the backhaul network is required.

4.1. Cell Edge MS Identification. Cell edge MSs are defined as MSs that have moderate SNR ( $>\mathrm{SNR}_{\text {threshold }}$ ) but low SIR ( $\leq$ SIR $_{\text {threshold }}$ ) values. MSs with low SNRs are noise limited. For noise limited MSs, mitigating the interference will increase the SINR slightly and these MSs can be better 
served by boosting the BS DL transmit power. Users that can receive signals from multiple BSs can measure the power level of the preambles transmitted by these BSs. IEEE 802.16e allows users to report their interference measurements to their serving BS for handover purposes.

After receiving MS interference reports, a BS determines which MSs would be cell edge MSs. The BS will report its total number of cell edge MSs to a RRM (radio resource management) unit over the backhaul. A BS only needs to update this information to the RRM when the number of its cell edge MSs changes significantly.

4.2. Resource Allocation for Cell Edge MSs. Upon receiving the number of cell edge users from all its BSs, the RRM unit determines how much resource to allocate across all BSs to serve cell edge MSs. For proportional fair type schedulers, the fraction of resource allocated to each MS in the long run is equal. Hence, we can allocate resources serving cell edge MSs to be proportional to the fraction of cell edge MSs present in the system. Let there be $Q$ scheduling quanta available per frame, the number of scheduling quanta allocated to serving cell edge MSs only $(U)$ can be determined as

$$
U=\left\lfloor Q \times \frac{\text { number of cell edge MSs }}{\text { total number of MSs }}\right\rfloor .
$$

The RRM unit allocates $U$ scheduling quanta to serve cell edge MSs and sends the size and location of the allocation to all BSs. Again, this allocation only needs to be updated when the ratio $U$ changes significantly.

4.3. Distributed Scheduling. Each BS schedules its cell edge MSs over the allocated resources in a distributed fashion with no coordination with other BSs. Conventional beamforming and nulling schemes proposed the restriction of equal UL and DL resource allocation. At the UL, each BS can calculate an approximation to the covariance matrix as $\widehat{\mathbf{R}}_{\mathbf{x x}_{i}}$ from the UL receive signal and use it to calculate the UL beamforming weight as in (4) and the DL beamforming weight as in (13). To eliminate the restriction of equal UL and DL resource allocation and still be able to calculate the covariance matrix estimation $\hat{\mathbf{R}}_{\mathbf{x x}_{i}}$ and calculate the UL/DL beamforming weight, we propose a special uplink sounding mechanism for our BFaN scheme in the next section.

4.4. Special Uplink Sounding. Special uplink sounding is used by BSs to estimate the covariance matrices and calculate the UL/DL beamforming weight while eliminating the equal UL and DL resource allocation restriction. This sounding design enables each BS to estimate its covariance matrix $\mathbf{R}_{\mathbf{x x}}$ which is needed in calculating beamforming weight as shown in (4) and (13).

Cell edge MSs that are allocated same resources in DL perform UL sounding in the UL using the same resources. This enables the BSs to estimate the covariance matrices. The UL sounding zone allocation spans the same frequency range as the cell edge user resource allocations in the DL. This ensures that the BF weight calculation at each BS takes into account the channel selectivity over the DL frequency allocation. In addition, this special sounding should be performed in the UL subframe right before the DL subframe to minimize the channel difference between the UL (when the BF weights are calculated) and DL (when the BF weights are applied).

An example resource allocation is depicted in Figure 5 to illustrate how our special UL sounding design works. In Figure 5, there are 8 resource blocks that are allocated to serve cell edge MSs. The 8 blocks are spread out over 2 time units and 4 frequency units. Hence, 8 sounding regions (SR) are required in the special sounding zone to estimate covariance matrices for the 8 resource blocks (RB). First the special uplink sounding blocks span the same 4 frequency units as the resource blocks. Next, subcarriers within each frequency unit in the special sounding region are divided into two distinct sets (dotted line and solid line subcarriers) to support the sounding for the resource blocks in the two time units. Hence, there are 8 sounding regions. Current uplink sounding allocation in 802.16 e supports our special uplink sounding allocation.

In Figure 5, BS 1 allocates resource blocks 1, 5, and 2 to MS 1 and BS 2 allocates resource blocks 1, 2, and 6 to MS 2. Hence, MS 1 will perform sounding in sounding regions 1, 5, and 2 and MS 2 will perform sounding in sounding regions 1,2 , and 6 . After the special UL sounding, each BS should be able to calculate an approximation to the covariance matrix using the averaging scheme in Figure 4 and calculate the BS beamforming weights using (4) and (13).

\section{Simulation Results}

Link level simulation alone cannot accurately quantify the performance gain of an interference mitigation scheme. This is due to the fact that the actual interference present in the system is a function of the resource scheduling performed at each BS. Without the actual scheduling function, the actual interference environment cannot be captured and the performance gain cannot be measured. We implemented our $\mathrm{BFaN}$ scheme in a system level simulator developed at Intel and are compliant to the IEEE $802.16 \mathrm{~m}$ Evaluation Methodology Document [11]. System level simulations with a proportional fair scheduler and full buffer traffic model at both the BSs and MSs are performed to investigate the system throughput improvement of our $\mathrm{BFaN}$ scheme. To reduce cost and power consumption at MSs, it is assumed that each MS uses 1 transmit in the UL and 2 receive antennae in the DL. A summary of simulation parameters can be found in Table 1.

To highlight the performance gain of our BFaN scheme, we compare the simulation result against a baseline system proposed in [11]. This baseline system is chosen due to the fact that all interference mitigation schemes proposed to the WiMAX standard compare their results to this baseline system. Hence, we can indirectly compare the performance of our scheme to all interference mitigation schemes in WiMAX if we use the same baseline system to showcase our results. In the baseline system, we switch between $2 \times 2$ STBC (Alamouti) or MIMO spatial multiplexing (SM) depending on channel conditions. In simulating the baseline system, 


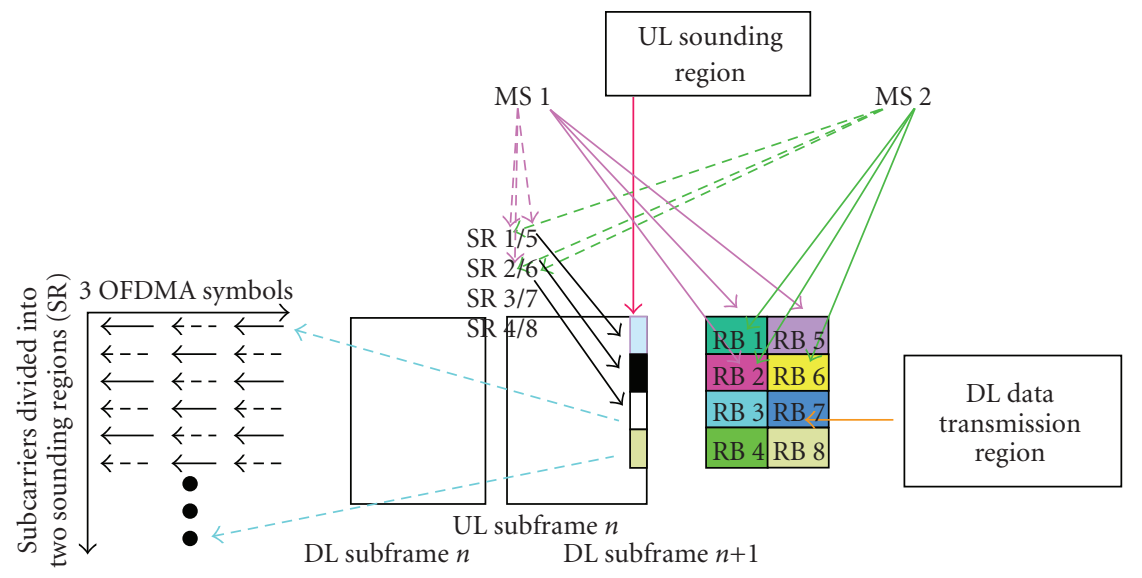

Figure 5: Special UL sounding design.

TABLE 1: Simulation parameters summary.

\begin{tabular}{ll}
\hline Parameters & Values \\
\hline Cellular layout & 7 cells or 19 cells, 3 sectors per cell \\
Inter-site distance & $1500 \mathrm{~m}$ \\
Fading channel & IEEE $802.16 \mathrm{~m}$ ITU-PEDB and IEEE \\
& $802.16 \mathrm{~m}$ ITU-VEHA \\
MS speed & $3 \mathrm{~km} / \mathrm{h}, 10 \mathrm{~km} / \mathrm{h}, 15 \mathrm{~km} / \mathrm{h}, 30 \mathrm{~km} / \mathrm{h}$, \\
& $60 \mathrm{~km} / \mathrm{h}$ \\
BFaNdwidth & $10 \mathrm{MHz}$ \\
Number of MS per sector & 10 \\
Subframe duration & $2.5 \mathrm{~ms}$ \\
& $2 \times 2(2 \mathrm{BS}$ Tx antennae, $2 \mathrm{MS} \mathrm{Rx}$ \\
Antenna configuration & antennae) baseline, $4 \times 2(4 \mathrm{BS}$ Tx \\
& antennas, $2 \mathrm{MS}$ Rx antennas) for \\
& our BFaN \\
\hline
\end{tabular}

delayed perfect channel knowledge is assumed. This implies that BSs use perfect channel that exists $5 \mathrm{~ms}$ ago to calculate the scheduling metrics and make scheduling decisions. When our BFaN scheme is enabled, BSs will use DL transmit beamforming to communication with the cell edge users using 2 or 4 BS antennas while cell center MSs are served using $2 \times 2 \mathrm{STBC} / \mathrm{SM}$ scheme.

5.1. Simulation Results and Comparison at Low Mobility. Simulation results at $3 \mathrm{~km} / \mathrm{h}$ with 19 cells are presented in Table 2. A simple rule of thumb [12] shows that for $M \times N$ antenna configuration, our BFaN can null out $M+N-2$ significant BS interferers. At the BS, it can form $M-1$ nulls and point them to $M-1$ cell edge MSs served by other BSs. This implies that the total number of interfering BSs experienced by any cell edge MS is reduced by $M-1$. At the MS, it can form $N-1$ nulls and point them to interfering BSs that do not point a null to the MS. Hence, using our BFaN at all BSs, the number of interfering BSs seen at a cell edge MS is reduced by $M+N-2$.

For $2 \times 2$ antenna configuration, our BFaN scheme can null out only 2 significant BS interferers. In a system with
TABLE 2: Simulation result from BFaN and PMI restriction schemes.

\begin{tabular}{lcc}
\hline Mode/performance & $\begin{array}{c}\text { Average MS } \\
\text { throughput increase } \\
\text { \% over baseline }\end{array}$ & $\begin{array}{c}\text { Cell edge MS } \\
\text { throughput increase } \\
\text { \% over baseline }\end{array}$ \\
\hline $2 \times 2$, reference scheme & 15.97 & 34.38 \\
$2 \times 2$, BFaN scheme & 9.69 & 15.63 \\
$2 \times 2$, PMI restriction & 1.9 & 39.8 \\
$4 \times 2$, reference scheme & 43.91 & 150 \\
$4 \times 2$, BFaN scheme & 19.17 & 68.75 \\
$4 \times 2$, PMI restriction & -0.5 & 32 \\
\hline
\end{tabular}

frequency reuse 1 , it is highly probable to find 3 or more significant BS interferers. Hence, our BFaN deliver modest gain at the cell edge without reducing cell center throughput rate as compared to the baseline system. In contrast, the PMI restriction promotes cell edge performance but sacrifices the cell center performance. In sum, our $\mathrm{BFaN}$ outperforms the PMI restriction at overall cell throughput but has a smaller cell edge improvement than the PMI restriction.

For $4 \times 2$ antenna configuration, our $\mathrm{BFaN}$ can null out approximately 4 significant BS interferers. With high probability, most significant interferers can be cancelled and high throughput rate is achieved by our $4 \times 2$ systems. Simulations show that our $4 \times 2$ scheme outperforms the PMI restriction in both the average user throughput rate and cell edge user throughput rate. A CDF of average MS throughput rate can be found in Figure 6. Cell edge user throughput rate is defined to be the throughput rate at $5 \%$ of the CDF curve. Average throughput rate is defined to be the throughput rate at $50 \%$ of the CDF curve.

The $4 \times 2$ reference scheme outperforms our $4 \times 2 \mathrm{BFaN}$ scheme by $81 \%$ at cell edge user throughput. This is due to the fact that the covariance matrix calculation in reference scheme is performed using perfect (but delayed) channel information. The covariance matrix calculation in our $\mathrm{BFaN}$ design is performed using UL sounding and can suffer from large cross terms (see the appendix) and approximation errors due to thermal noise. The effect of the cross terms can 


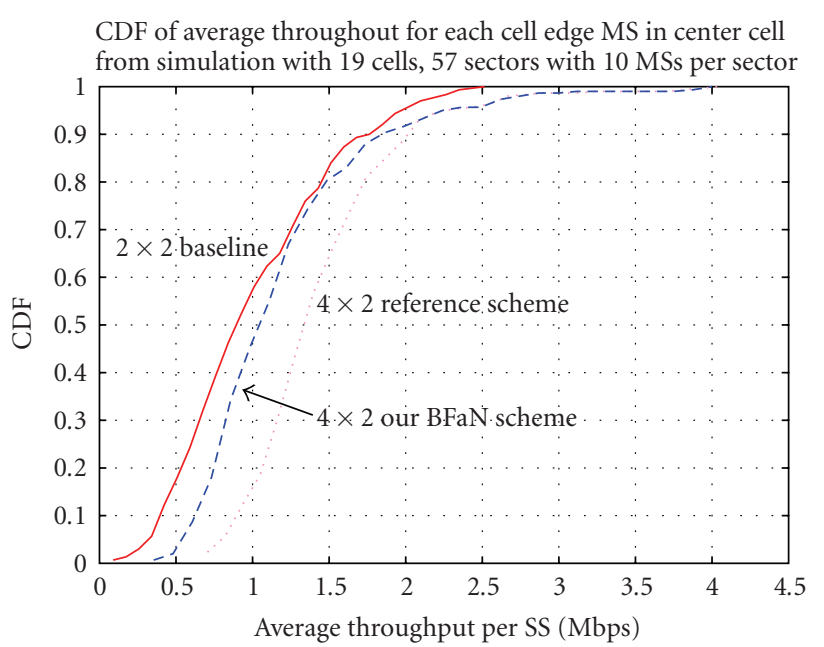

Figure 6: CDF of average MS throughput rate of BFaN.

be reduced by expanding the number of subcarriers in the averaging. However, this increases the system overhead.

5.2. Simulation Results for Higher Mobile Speeds. As discussed previously, feedback and computation delays of $5 \mathrm{~ms}$ is introduced between the time when beamforming weights are calculated and when the beamforming weights are actually applied. This causes channel mismatch (which increases with mobile speeds) between the time the BS beamforming weights are calculated to the time when BS beamforming weights are applied. From Figure 7, it is observed that gain in cell edge MS throughput rate drops as MS speed increases. However, if MS speed is kept under $60 \mathrm{~km} / \mathrm{h}$ per hour, our $\mathrm{BFaN}$ scheme can still provide gain over the baseline system.

There are two other interesting observations. First, the reference scheme is more sensitive to the channel mismatch caused by user mobility than our BFaN scheme. This is due to the fact that the $\mathrm{BFaN}$ scheme forms wider beams and shallower nulls than the reference design. Hence, the channel mismatch causes greater error in the beamforming and null forming and pointing in the reference scheme at higher mobile speed and degrade the system performance more. Second, as the mobile speed increases, performance of the baseline system drops more than our BFaN schemes at moderate MS speed upto $30 \mathrm{~km} / \mathrm{h}$. It is only at higher MS speeds that our BFaN schemes start to drop more in system performance compared to the baseline system.

\section{Conclusions}

The optimality and efficiency of our BFaN scheme is proved and quantified by derivation and system level simulations, respectively. It increases the cell edge MS throughput rate compared to the baseline implementation upto MS speed of $60 \mathrm{~km} / \mathrm{h}$. Our special uplink sounding provides an elegant way to enable UL/DL BS beamforming weight calculation and eliminates the restriction of equal UL and DL resource allocation and real time information exchange over the

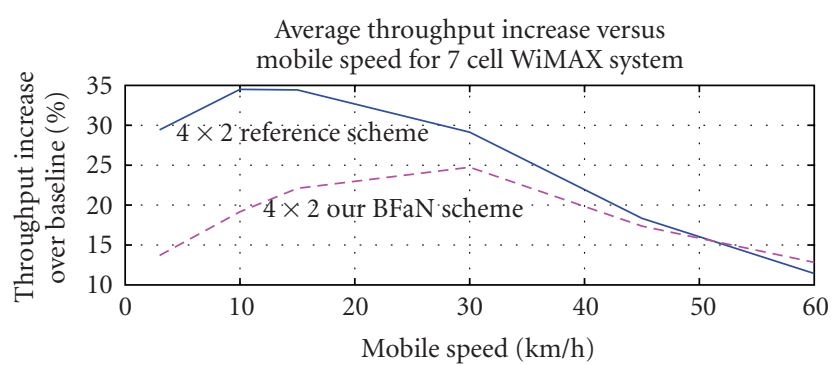

(a)

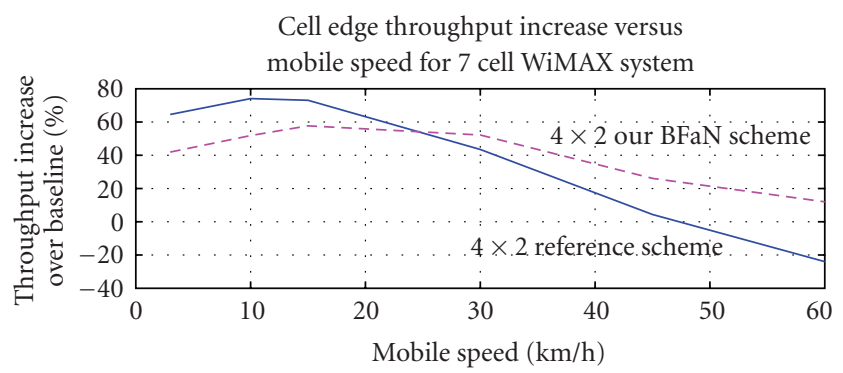

(b)

FIGURE 7: Throughput rate increase of BFaN over the baseline system with moving users.

backhaul. A survey of recent schemes for interference mitigation is presented and one of them is compared to our proposed scheme. Our scheme is simple to implement since it is already supported by the current $802.16 \mathrm{e}$ message exchange and sounding mechanisms.

\section{Appendix}

Assuming channel is flat over $K$ subcarriers and $T$ symbols, covariance matrix $\hat{\mathbf{R}}_{\mathbf{x x}_{i}}(k)$ is expanded as

$$
\begin{aligned}
\hat{\mathbf{R}}_{\mathbf{x x}}= & \frac{1}{K \cdot T} \sum_{k=l, t=m}^{l+K-1, m+T-1} \mathbf{x}_{i} \mathbf{x}_{i}^{H} \\
= & \mathbf{H}_{i}^{T} \mathbf{w}_{\mathrm{MS} i} \mathbf{w}_{\mathrm{MS} i}^{H}\left(\mathbf{H}_{i}^{T}\right)^{H} \frac{1}{K \cdot T} \\
& \times \sum_{k=l, t=m}^{l+K-1, m+T-1} s_{i}(k, t) s_{i}^{*}(k, t) \\
& +\sum_{j, i \neq j, \text { celledge users of }} \mathbf{G}_{i j}^{T} \mathbf{w}_{\mathrm{MS} j} \mathbf{w}_{\mathrm{MS} j}^{H}\left(\mathbf{G}_{i}^{T}\right)^{H} \frac{1}{K \cdot T} \\
& \times \sum_{k=l, t=m}^{l+K-1, m+T-1} s_{j}(k, t) s_{j}^{*}(k, t) \\
& +\frac{1}{K \cdot T} \sum_{k=l, t=m}^{l+K-1, m+T-1} \mathbf{n}(k, t)(\mathbf{n}(k, t))^{H}
\end{aligned}
$$

(needed terms) 


$$
\begin{aligned}
& +\mathbf{H}_{i}^{T} \mathbf{w}_{\mathrm{MSi}}\left(\sum_{\substack{j, i \neq j, \text { cell edge users of } \\
\text { all interfering BS }}} \mathbf{w}_{\mathrm{MS} j}^{H}\left(\mathbf{G}_{i j}^{T}\right)^{H} \frac{1}{K \cdot T}\right. \\
& \times \sum_{k=l, t=m}^{l+K-1, m+T-1} s_{i}(k, t) s_{j}^{*}(k, t) \\
& \left.+\frac{1}{K \cdot T} \sum_{k=l, t=m}^{l+K-1, m+T-1} s_{i}(k, t)(\mathbf{n}(k, t))^{H}\right) \\
& +\sum_{j, i \neq j, \text { cell edge users of }} \mathbf{G}_{i j}^{T} \mathbf{w}_{\mathrm{MS} j} \mathbf{w}_{\mathrm{MS} j}^{H}\left(\mathbf{H}_{i}^{T}\right)^{H} \frac{1}{K \cdot T} \\
& \text { all interfering BSs } \\
& \times \sum_{k=l, t=m}^{l+K-1, m+T-1} s_{j}(k, t)\left(s_{i}^{*}(k, t)+(\mathbf{n}(k, t))^{H}\right) \\
& +\frac{1}{K \cdot T} \sum_{k=l, t=m}^{l+K-1, m+T-1} \mathbf{n}(k, t) \\
& \times\left(s_{i}^{*}(k, t) \mathbf{w}_{\mathrm{MS} i}^{H}\left(\mathbf{H}_{i}^{T}\right)^{H}+s_{j}^{*}(k, t)\right. \\
& \left.\times \sum_{\substack{j, i \neq j \text {, cell edge users of } \\
\text { all interfering BSs }}} \mathbf{w}_{\mathrm{MS} j}^{H}\left(\mathbf{G}_{i j}^{T}\right)^{H}\right)
\end{aligned}
$$

(cross-terms).
[6] J. Kim, D. Lim, W. Lee, and B.-C. Ihm, "PMI restriction with adaptive feedback mode," IEEE C802.16m-09/0023, January 2009.

[7] S. Kim, J. Kim, D. Lim, B.-C. Ihm, and H. Cho, "Interference mitigation using FFR and multi-cell MIMO in downlink," IEEE C802.16m-08/783, July 2008.

[8] I. Emre Telatar, "Capacity of multi-antenna gaussian channels," in Proceedings IEEE International Symposium on Information Theory, 2001.

[9] A. Maltsev, R. Maslennikov, and A. Khoryaev, "Comparative analysis of spatial covariance matrix estimation methods in OFDM communication systems," in Proceedings of the 6th IEEE International Symposium on Signal Processing andInformation Technology (ISSPIT '06), pp. 551-555, August 2006.

[10] Q. Li, J. Zhu, Q. Li, and C. Georghiades, "Efficient spatial covariance estimation for asynchronous co-channel interference suppression in MIMO-OFDM systems," IEEE Transactions on Wireless Communications, vol. 7, no. 12, pp. 4849$4853,2008$.

[11] IEEE 802.16m-08/004r5, "Project 802.16m Evaluation Methodology Document (EMD),” January 2009.

[12] L. C. Godara, "Applications of antenna arrays to mobile communications_-part I: performance improvement, feasibility, and system considerations," Proceedings of the IEEE, vol. 85, no. 7, pp. 1031-1060, 1997.

\section{References}

[1] Q. Li, X. E. Lin, and J. Zhang, "MIMO precoding in 802.16e WiMAX," Journal of Communications and Networks, vol. 9, no. 2, pp. 141-149, 2007.

[2] P. Viswanath, D. N. C. Tse, and R. Laroia, "Opportunistic beamforming using dumb antennas," IEEE Transactions on Information Theory, vol. 48, no. 6, pp. 1277-1294, 2002.

[3] L. C. Godara, "Application of antenna arrays to mobile communications-part II: beam-forming and direction-ofarrival considerations," Proceedings of the IEEE, vol. 85, no. 8, pp. 1195-1245, 1997.

[4] S. Hamiti, Nokia, and SDD, Eds., "IEEE $802.16 \mathrm{~m}$ system description document [Draft]," IEEE $802.16 \mathrm{~m}-08 / 003 \mathrm{r} 9 \mathrm{a}$, 2009-05-31.

[5] D. Lim, S. Kim, J. Kim, and B.-C. Ihm, "PMI restriction for the downlink closed-loop MIMO,” IEEE C802.16m-08_430r1.doc, July 2008. 

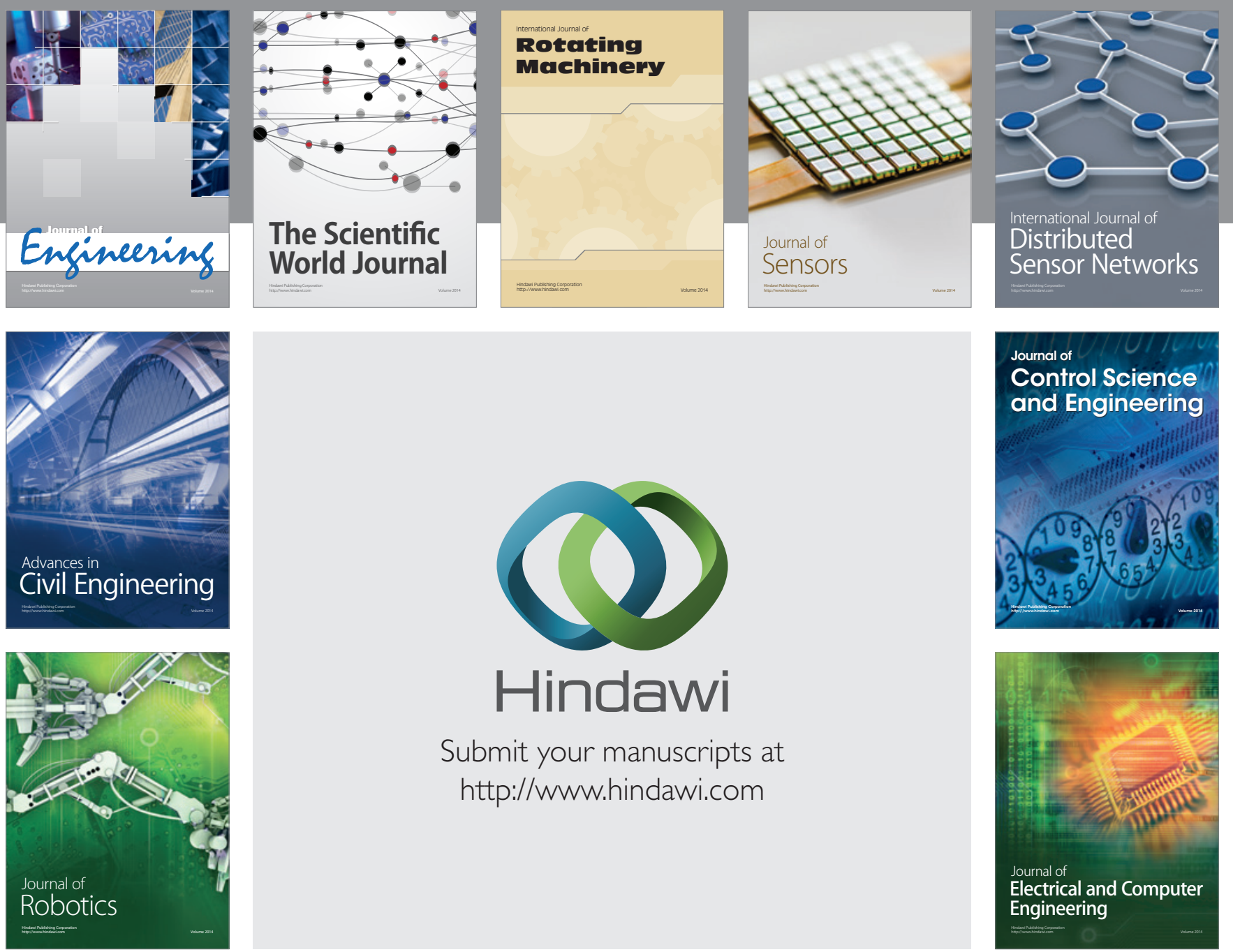

Submit your manuscripts at

http://www.hindawi.com
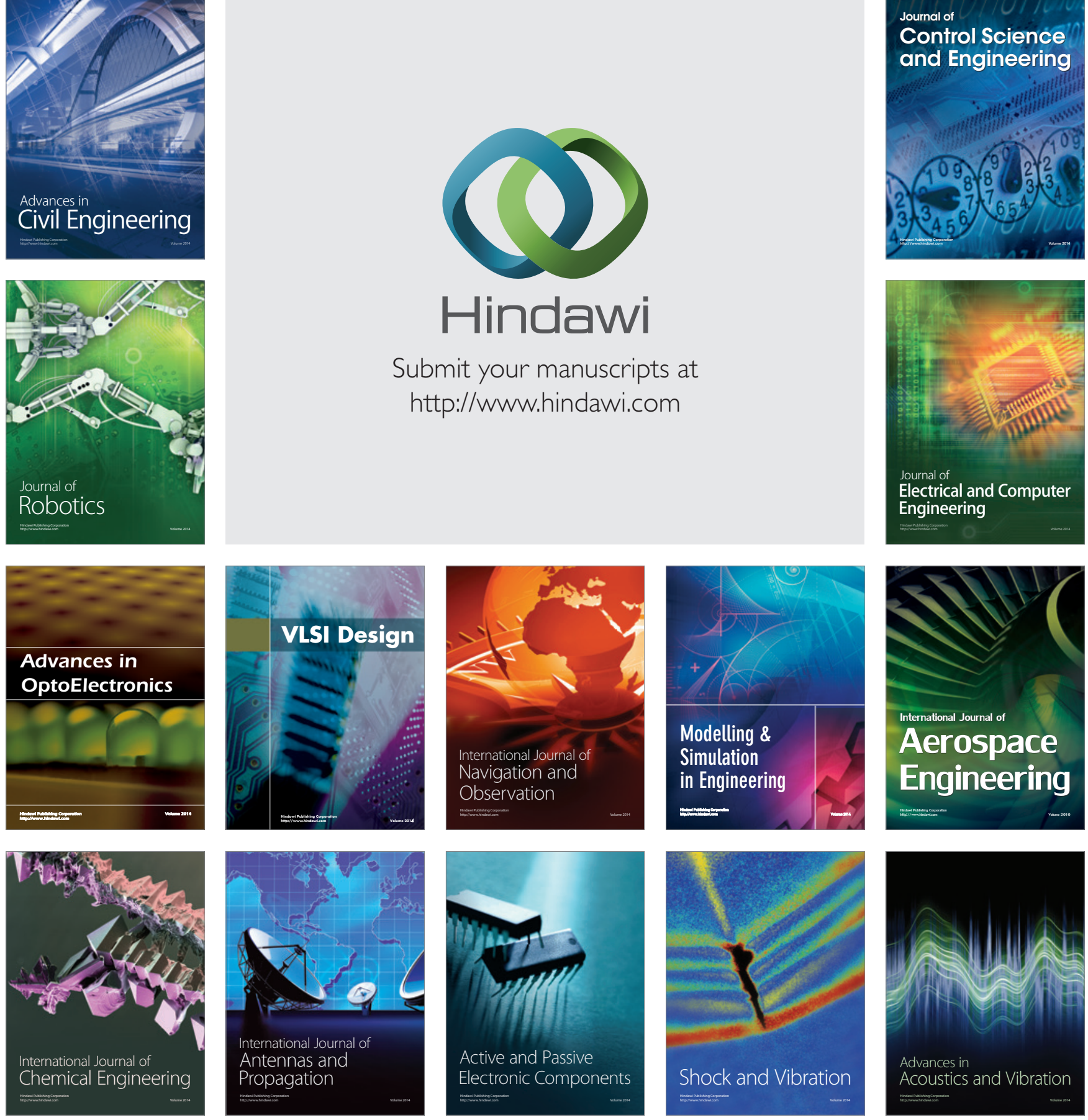